\title{
Light speed rotating black holes: The special holes
}

\author{
U. V.S. Seshavatharam ${ }^{* 1,2}$ \\ ${ }^{1}$ Honorary faculty, I-SERVE, Alakapuri, Hyderabad-35, AP, India \\ ${ }^{2}$ Sr. Engineer, QA-Spun, Lanco Industries Ltd, Srikalahasti-517641, AP, India \\ *Corresponding author E-mail: seshavatharam.uvs@gmail.com
}

\begin{abstract}
Astronomers believe that black holes that are as large as a billion solar masses can be found at the centre of most galaxies. Here the fundamental questions to be answered are: If the galactic central black hole mass is 10 billion solar masses and density is less than $1 \mathrm{~kg} / \mathrm{m}^{3}$ - with such a small density and large mass, without collapsing - how it is able to hold a gigantic galaxy? What force makes the black hole stable? Recent observations indicate that, instead of collapsing, galactic central black holes are growing faster and spinning faster than they expected. From this it can be suggested that, in the universe - 'black hole geometry' is most important than its mass and density. In this regard, considering the classical force and power limits an attempt is made to understand the black hole geometry, radius, angular velocity and temperature. If one is willing to consider the 'black hole geometry' as primordial, if universe constitutes so many galaxies, if each galaxy constitutes a central fast growing and fast spinning black hole and if black hole's growth accelerates with its increasing mass, then considering universe as a 'growing and light speed rotating primordial black hole' may not be far away from reality. May be the vastness of the universe making our observations limited and forcing us to believe that - universe is flat. Thinking in this way and with reference to the Planck scale an attempt is made to fit the present Hubble's constant and its obtained value is $66.895(\mathrm{~km} / \mathrm{sec}) / \mathrm{Mpc}$. It can be compared with the recently recommended value $(67.80 \pm 0.77)(\mathrm{km} / \mathrm{sec}) / \mathrm{Mpc}$
\end{abstract}

Keywords: Classical force limit; Classical power limit; Light speed rotating black holes - the special holes; Hawking-Unruh temperature; Cosmic geometry; Hubble's constant;

\section{Introduction}

A black hole is a place where space - time takes maximum possible curvature and hence gravity is so strong. Black holes are such a fascinating topic of physics that they have fascinated both young and old, since they have come into light. Incidentally, the name 'black hole' was invented by John Archibald Wheeler, and seems to have stuck because it was much catchier than previous names. Before Wheeler came along, these objects were often referred to as 'frozen stars'. The idea of a mass concentration so dense that even light would be trapped goes all the way back to Laplace in the 18th century. Almost immediately after Einstein developed general relativity, Karl Schwarzschild discovered a mathematical solution to the equations of the theory that described such an object. It was only much later, with the work of such people as Oppenheimer, Volkoff, and Snyder in the 1930's, that people thought seriously about the possibility that such objects might actually exist in the Universe. These researchers showed that when a sufficiently massive star runs out of fuel, it is unable to support itself against its own gravitational pull, and it should collapse into a black hole[1,2].

Considering the exotic nature of black holes, it may be natural to question if such bizarre objects could exist in nature or to suggest that they are merely pathological solutions to Einstein's equations. Einstein himself wrongly thought that black holes would not form, because he held that the angular momentum of collapsing particles would stabilize their motion at some radius. This led the general relativity community to dismiss all results to the contrary for many years. However, a minority of relativists continued to contend that black holes were physical objects, and by the end of the 1960s, they had persuaded the majority of researchers in the field that there is no obstacle to forming an event horizon. Modern cosmologists are deeply involved in understanding the basic physical identity of 'dark matter' and 'dark energy'. At this juncture, staring from micro black holes to the super massive black holes, it is futile to comment about the basic constituents of a black hole and events that may happen inside the black hole. Whether a black hole is the end point of matter or it is the base point of observable cosmic matter - is still a mystery in the current physics world. Considering the classical limits of force and power $[3,4]$ and rest energy of black holes, in this paper an attempt is made 
to understand the light speed rotating black holes, their minimum size, maximum angular velocity and maximum temperature.

\section{Mass limits, formation and growth of a Black hole}

There is no perfect theory that defines the lower and upper limits of a massive black hole. As the radius of a black hole is directly proportional to its mass, its density decreases as its mass increases. If mass is at lower side, density will be very high, if mass is higher side, density will be very low. If mass of black hole is $10^{40} \mathrm{~kg}$, then black hole's density will be close to $0.73 \mathrm{Kg} / \mathrm{m}^{3}$ and is less than the air density. The fundamental question to be understood is: if black hole mass is too high and density is too less, inside a black hole whether our known physical laws will work? Answer is beyond the scope of current physics.

Generally it is believed that, black holes are formed by gravitational collapse of massive stars. Gravitational collapse requires great density. In the current epoch of the universe these high densities are only found in stars, but in the early universe shortly after the big bang densities were much greater, possibly allowing for the creation of black holes. The high density alone is not enough to allow the formation of black holes since a uniform mass distribution will not allow the mass to bunch up. In order for primordial black hole to form in such a dense medium, there must be initial density perturbations that can then grow under their own gravity. Different models for the early universe vary widely in their predictions of the size of these perturbations. Various models predict the creation of black holes, ranging from a Planck mass to hundreds of thousands of solar masses. Primordial black holes could thus account for the creation of any type of black hole.

Once a black hole has been formed, it can continue to grow by absorbing additional matter. Any black hole will continually absorb gas and interstellar dust from its direct surroundings and omnipresent cosmic background radiation. This is the primary process through which super massive black holes seem to have grown[5]. A similar process has been suggested for the formation of intermediate-mass black holes in globular clusters. Another possibility is for a black hole to merge with other objects such as stars or even other black holes. Although not necessary for growth, this is thought to have been important, especially for the early development of super massive black holes, which could have formed from the coagulation of many smaller objects. The process has also been proposed as the origin of some intermediate-mass black holes. Astronomers believe that black holes that are as large as a billion solar masses can be found at the centre of most galaxies. Here the fundamental questions to be answered are: If the galactic central black hole mass is 10 billion solar masses and density is less than $1 \mathrm{~kg} / \mathrm{m}^{3}$ - with such a small density and large mass, without collapsing- how it is able to hold a gigantic galaxy? What force makes the black hole stable?

Gravitational collapse is not the only process that could create black holes. In principle, black holes could be formed in high-energy collisions that achieve sufficient density. As of 2012, no such events have been detected, either directly or indirectly as a deficiency of the mass balance in particle accelerator experiments. This suggests that there must be a lower limit for the mass of black holes. Theoretically, this boundary is expected to lie around the Planck mass where quantum effects are expected to invalidate the predictions of general relativity. Note that scientists are expecting the formation of micro black holes in high energy collisions.

\section{Rotating black holes: Black hole found spinning near the relativistic limit}

The primary question is: Do black holes exist? If the answer is yes, then spinning black holes are inevitable, since astronomical bodies most often rotate. A rotating black hole is a solution of Einstein's field equation. There are two known exact solutions, the Kerr metric and the Kerr-Newman metric, which are believed to be representative of all rotating black hole solutions, in the exterior region. Astrophysicists believe that studies of super massive black hole's spin in galaxies throughout the universe will provide important information about the formation and evolution of galaxies. In 2013 February, using Nasa's newly launched NuStar telescope and the European Space Agency's workhorse XMM-Newton, an international team observed high-energy X-rays released by a super massive black hole in the middle of a nearby galaxy. They calculated its spin at close to the speed of light: 670m mph [6]. In late 2006, astronomers reported estimates of the spin rates of black holes in the Astrophysical Journal. A black hole in the Milky Way, GRS 1915+105, may rotate 1,150 times per second, approaching the theoretical upper limit.

Rotating black holes are formed in the gravitational collapse of a massive spinning star or from the collapse of a collection of stars or gas with a total non-zero angular momentum. As most stars rotate it is expected that most black holes in nature are rotating black holes. The best evidence yet that some super massive black holes (SMBH) rotate at extremely high rates has been found by an international team of astronomers. Made using the recently launched NuStar space telescope, the study suggests that a huge black hole at the centre of a distant galaxy acquired a huge amount of rotational energy as it formed. The discovery could provide important information about how SMBHs and their 
associated galaxies form and evolve. Knowing the spin of an SMBH can provide important clues about how it formed. If the black hole grew slowly, by sucking in small amounts of matter from all directions, then it isn't expected to have much spin. However, if the formation process involves the black hole gorging rapidly on matter from a specific direction, conservation of angular momentum would leave it with an extremely large spin. The spin of a super massive black hole can be measured by looking at the effect that the spin has on material that is being sucked in to the black hole. This material forms an accretion disc that swirls around the black hole before disappearing from sight. The faster the black hole is spinning, the closer the inner edge of the disc is to the centre of the black hole. As a result, the X-rays emanating from the inner edge are affected by the black hole's gravity more when the black hole is spinning.

\section{Classical limits of force and power}

It is accepted that a complete theory of the evolution of the universe will not be possible until the development of a successful quantum theory of gravity. The outstanding problem in particle physics today is the inclusion of gravity in a single, unified quantum theory of all the fundamental interactions. Particle physicists have long suggested that the four fundamental forces of nature (viz. the gravitational, electromagnetic, weak nuclear and strong nuclear forces) are separate, low energy manifestations of what was once a single force at times close to the Big Bang. It is postulated that as the universe expanded and cooled, this single force gradually broke down into the four separate interactions observed today. A detailed quantum theory that describes the electromagnetic and weak nuclear forces in terms of a single force (the electroweak interaction) was developed in the 1970's and dramatically verified by high-energy experiments in particle accelerators a decade later. More ambitious quantum theories that incorporate the strong nuclear force (Grand Unified Theories) have been developed, and some experimental support for these models has emerged. However, unification theories that seek to unify the force of gravity with all the other forces (Theories of Everything) remain elusive, as the gravitational interaction lacks a quantum formulation.

To unify cosmology, quantum mechanics and the four observed fundamental cosmological interactions - certainly a 'unified force' is required. In this connection $\left(c^{4} / G\right)$ can be considered as the classical force limit $[3,4]$. Similarly $\left(c^{5} / G\right)$ can be considered as the classical power limit. If it is true that $c$ and $G$ are fundamental physical constants in physics, then $\left(c^{4} / G\right)$ and $\left(c^{5} / G\right)$ can also be considered as fundamental compound physical constants. These limits are more powerful than the Uncertainty limit.

Simple applications of $\left(c^{4} / G\right)$ can be stated as follows

1. Magnitude of force of attraction or repulsion between any two charged particles never crosses $\left(c^{4} / G\right)$.

2. Magnitude of gravitational force of attraction between any two massive bodies never crosses $\left(c^{4} / G\right)$.

3. Magnitude of mechanical force on a revolving/rotating body never crosses $\left(c^{4} / G\right)$.

4. Magnitude of electromagnetic force on a revolving body never crosses $\left(c^{4} / G\right)$.

Simple applications of $\left(c^{5} / G\right)$ can be stated as follows

1. Mechanical power (either input or output) never crosses $\left(c^{5} / G\right)$

2. Electromagnetic power (either input or output) never crosses $\left(c^{5} / G\right)$

3. Thermal radiation power (either input or output) never crosses $\left(c^{5} / G\right)$

4. Gravitational radiation power (either input or output) never crosses $\left(c^{5} / G\right)$ 


\section{Light speed rotating Black Holes: The special holes}

The 4 basic physical properties of a rotating black hole are its mass, size, angular velocity and temperature. Without going deep into the mathematics of black hole physics in the following subsections an attempt is made to understand and fit the black hole radius and temperature.

\subsection{Rotating black hole radius}

As a single body problem, rotating black holes can be understood with the classical force limit $\left(c^{4} / G\right)$, classical power limit $\left(c^{5} / G\right)$ and $\left(M c^{2}\right)$ within 3 to 4 steps. For any rotating celestial body assume that,

torque, $\tau \leq M c^{2}$

power, $P=\tau \omega \leq \frac{c^{5}}{G}$

thus, $\omega \leq \frac{\mathrm{c}^{3}}{\mathrm{GM}}$ and $\omega_{\max }=\frac{\mathrm{c}^{3}}{\mathrm{GM}}$

If the celestial body or black hole rotates at light speed, to have maximum angular velocity, radius should be minimum and can be expressed as:

$R_{\min }=\frac{c}{\omega_{\max }}=\frac{G M}{c^{2}} \cong \frac{M c^{2}}{\left(c^{4} / G\right)}$

This expression is similar to the 'Schwarzschild radius' of a black hole. The only change is that coefficient 2 is missing. This is really a very interesting case. This obtained expression indicates that, to get 'light speed rotation', celestial body should have a 'minimum radius' of $G M / c^{2}$. Logically the Schwarzschild radius can be expressed as follows. If no force (of zero magnitude) acts on the mass content $M$ of the assumed massive body, from relation (4) size of the massive body approaches infinity. If a force of magnitude $\left(c^{4} / G\right)$ acts on the mass content of the assumed body it approaches a minimum radius of $\left(G M / c^{2}\right)$. With reference to the average magnitude $\left(0+\frac{c^{4}}{G}\right) \cong \frac{c^{4}}{2 G}$, the presently believed Schwarzschild radius can be obtained as

$(R)_{\text {ave }} \cong \frac{M c^{2}}{\left(c^{4} / 2 G\right)} \cong \frac{2 G M}{c^{2}}$

Clearly speaking this proposal is very simple and is entirely different from the existing concepts of General theory of relativity. It is not speaking about the gravitational collapse of stars or singularity. If the concept of 'Schwarzschild radius' is believed to be true, for any rotating celestial body or black hole of rest mass $M$ the critical conditions can be stated as follows.

\section{Case-1: Two statements}

1) Magnitude of 'torque' never crosses 'rest energy' .

2) Magnitude of mechanical power never crosses $\frac{1}{2}\left(c^{5} / G\right)$.

Now above relations (1) to (4) can be re-expressed as follows.

torque, $\tau \leq M c^{2}$

power, $P=\tau \omega \leq \frac{c^{5}}{2 G}$

thus, $\omega \leq \frac{\mathrm{c}^{3}}{2 \mathrm{GM}}$ and $\omega_{\max }=\frac{\mathrm{c}^{3}}{2 \mathrm{GM}}$

When the celestial body rotates at light speed, to have maximum angular velocity, radius should be minimum as,

$R_{\text {min }}=\frac{c}{\omega_{\max }}=\frac{2 G M}{c^{2}}$ 
Case-2: Four statements

1) Magnitude of 'kinetic energy' never crosses 'rest energy'.

2) Magnitude of 'torque' never crosses 'potential energy' and

3) Magnitude of mechanical power never crosses $\left(c^{5} / G\right)$.

4) Note that, based on the Virial theorem, potential energy is twice of kinetic energy and thus,

$$
\tau \leq 2 M c^{2} \text {. }
$$

In this way in a simplified way, existence of rotating black hole concepts can be understood. Now above relations (1) to (4) can be re-expressed as follows.

$$
\begin{aligned}
& \text { torque, } \tau \leq 2 M c^{2} \\
& \text { power, } P=\tau \omega \leq \frac{c^{5}}{G} \\
& \text { thus, } \omega \leq \frac{c^{3}}{2 \mathrm{GM}} \text { and } \omega_{\max }=\frac{c^{3}}{2 \mathrm{GM}}
\end{aligned}
$$

When the celestial body rotates at light speed, to have maximum angular velocity, radius should be minimum as,

$$
R_{\min }=\frac{c}{\omega_{\max }}=\frac{2 G M}{c^{2}}
$$

This expression is similar to the 'Schwarzschild radius' of a black hole and represents light speed rotation for any celestial sphere or black hole. Based on Newton's law of gravitation, acceleration due to gravity at the light speed rotating black hole or celestial body can be expressed as

$$
g_{\max } \cong \frac{G M}{R_{\min }^{2}} \cong \frac{c^{4}}{4 G M}
$$

\subsection{Rotating black hole angular velocity and temperature}

According to S.W.Hawking [7], black hole temperature can be expressed as

$$
T \cong \frac{\hbar c^{3}}{8 \pi G k_{B} M}
$$

It can be re-arranged as follows.

$$
4 \pi k_{B} T_{\max } \cong \frac{\hbar c^{3}}{2 G M} \cong \hbar\left(\frac{c^{3}}{2 G M}\right) \cong \hbar \omega_{\max }
$$

From this expression it can be suggested that, as the black hole angular velocity increases its temperature increases. When the black hole rotates at light speed, its angular velocity reaches to a maximum and hence temperature reaches to a maximum. Very interesting result is that, a stationary black hole cannot emit any thermal radiation and an accelerating black hole emits thermal radiation with increasing temperature. This concepts resembles the 'Unruh effect' [8]. The hypothetical Unruh effect is the prediction that an accelerating observer will observe black-body radiation where an inertial observer would observe none. In other words, the background appears to be warm from an accelerating reference frame; in layman's terms, a thermometer waved around in empty space will record a non-zero temperature. The ground state for an inertial observer is seen as in thermodynamic equilibrium with a non-zero temperature by the uniformly accelerated observer. It is currently not clear whether the Unruh effect has actually been observed, since the claimed observations are under dispute. There is also some doubt about whether the Unruh effect implies the existence of Unruh radiation. The Unruh temperature has the same form as the Hawking temperature of a black hole, which was derived by Stephen Hawking independently around the same time. It is, therefore, sometimes called the HawkingUnruh temperature.

\section{The cosmic geometry and the CMB radiation temperature}

\subsection{Major Shortcomings of Modern Cosmology}

A) If light is coming from the atomic matter of the galaxy, then redshift can be interpreted as an index of the galactic atomic matter 'light emission mechanism'. In no way it seems to be connected with 'galaxy receding' $[9,10]$. 
B) If cosmic expansion is continuous and accelerating and redshift is a measure of cosmic expansion, 'rate of increase in redshift' can be considered as a measure of cosmic 'rate of expansion'. Then there is no possibility to observe a 'constant' red shift. Merely by estimating galaxy distance (instead of estimating galaxy receding speed) one cannot verify the cosmic acceleration. More over the current definition of red shift seems to be ad-hoc and not absolute hence one may not be able to understand or confirm the actual cosmic rate of expansion[10].

C) Drop in cosmic temperature' can be considered as a measure of cosmic expansion and 'rate of decrease in cosmic temperature' can be considered as a measure of cosmic 'rate of expansion'. But if rate of decrease in temperature is very small and is beyond the scope of current experimental verification, then the two possible states are: a) cosmic temperature is decreasing at a very slow rate and universe is expanding at a very slow rate and b) there is no 'observable' thermal expansion and there is no 'observable' cosmic expansion.

D) If 'Dark energy' is the major outcome of the 'accelerating universe', it is very important to note that - in understanding the basic concepts of unification or other fundamental areas of physics, role of dark energy is very insignificant. So far no ground based experiment confirmed the existence of dark energy. There is no single clue or definition or evidence to any of the natural physical properties of (the assumed) dark energy.

E) Dimensionally it is possible to show that, the dimensions of Hubble's constant and angular velocity are same. If so considering Hubble's constant merely as an expansion parameter may not be correct.

\subsection{Black hole Cosmology or Schwarzschild Cosmology}

A black-hole cosmology (also called the Schwarzschild cosmology ) is a cosmological model in which the observable universe is the interior of a black hole. Such models were originally proposed by Raj Pathria [11], I. J. Good [12], Nikodem Popławski [13], Tinaxi Zhang [14], Joel Smoller, Blake Temple [15] and Chul-Moon Yoo [16]. Any such model requires that the Hubble radius of the observable universe is equal to its Schwarzschild radius, that is, the product of its mass and the Schwarzschild proportionality constant. This is indeed known to be nearly the case; however, most cosmologists consider this close match a 'coincidence'. In the version as originally proposed by Pathria and Good, and studied more recently by others can be stated as : the observable universe is the interior of a black hole existing inside a larger universe or multi-verse.

Now the primordial question to be answered is: which is primordial : either the black hole 'geometry' or the black hole 'mass'? Considering the concept of 'growing black holes' [17] - it is very easy to say that, the concept 'black hole geometry' is more primordial than the concept 'black hole mass'. If one is willing to consider the 'black hole geometry' as primordial, then considering the concept of growing black holes, entire universe can be considered as a primordial growing black hole $[3,4]$. Please note that

if universe constitutes so many galaxies, (well observed)

if each galaxy constitutes a central fast growing and fast spinning black hole, (well believed)

if black hole's growth accelerates with its increasing mass, (to be confirmed)

then considering universe as a fast spinning and fast growing black hole may not be far away from reality. This idea resembles the current notion of accelerating universe but differs from the flat model of the universe. Here one should note that there is one compromise in between the flat universe and the closed universe: Hubble volume - the observable universe! For the time being let us speculate that, right from the Planck scale, universe is a growing and light speed rotating primordial black hole. If $M_{P}$ is the

Planck mass, at Planck scale, cosmic angular velocity can be expressed as

$\left(\omega_{\max }\right)_{\text {Planck }} \cong \frac{c^{3}}{2 G M_{P}} \cong \frac{1}{2} \sqrt{\frac{c^{5}}{G \hbar}}$

At present also if universe is rotating at (a maximum speed) of light speed and the present Hubble's constant represents its maximum angular velocity, then

$\left(\omega_{\max }\right)_{\text {present }} \cong H_{0}$

Considering the current $\mathrm{CMB}$ radiation temperature [18], $T \cong 2.72548 \pm 0.00057 \mathrm{~K}$ as the maximum temperature of the current black hole universe and from relation (17), empirically to a very good accuracy it is noticed that,

$$
4 \pi k_{B} T_{\max } \cong \hbar \sqrt{\left(\omega_{\max }\right)_{\text {Planck }} \cdot\left(\omega_{\max }\right)_{\text {present }}} \cong \sqrt{\frac{\hbar}{2} \sqrt{\frac{\hbar c^{5}}{G}} \cdot H_{0}}
$$

Now $T_{\max }$ can be expressed as

$T_{\max } \cong \sqrt{\frac{\left(\omega_{\max }\right)_{\text {Planck }}}{\left(\omega_{\max }\right)_{\text {present }}}} \cdot \frac{\hbar\left(\omega_{\max }\right)_{\text {present }}}{4 \pi k_{B}} \cong \sqrt{\frac{\left(\omega_{\max }\right)_{\text {Planck }}}{H_{0}}} \cdot \frac{\hbar H_{0}}{4 \pi k_{B}}$ 
Thus the current Hubble's constant can be expressed as

$H_{0} \cong\left(4 \pi k_{B} T_{\text {max }}\right)^{2} \div\left(\frac{\hbar}{2} \sqrt{\frac{\hbar c^{5}}{G}}\right) \cong 2.168 \times 10^{-18} \mathrm{sec}^{-1} \cong 66.895(\mathrm{~km} / \mathrm{sec}) / \mathrm{Mpc}$

This obtained magnitude of $H_{0}$ can be compared with the recently recommend magnitude of the Hubble's constant: $(67.80 \pm 0.77)(\mathrm{km} / \mathrm{sec}) / \mathrm{Mpc}[18,19]$. Even though it seems to be an accidental coincidence, from unification point of view this coincidence can not be ignored. From geometry point of view - may be the vastness of the universe making our observations limited and forcing us to believe that - universe is flat. Even though 'dark energy' proposal is quite interesting, its physical identification seems to be far away from reality. At present, astrophysicists believe that recent observations of Super-Eddington accreting massive black holes at high redshift may provide some insight [20] on the cosmic rate of expansion. This idea directly and indirectly indicates the role of galactic central black holes in understanding the cosmic evolution. Similar to the chicken-egg problem, now the fundamental question to be answered is : which is a primordial black hole: either the universe or galaxy centre?

\section{Conclusion}

In the universe - 'black hole geometry' or 'black hole structure' can be given more importance than black hole's 'growing mass' or 'decreasing density'. It can be suggested that, any celestial body or black hole of mass $M$ can have light speed rotation if its radius approaches $\left(2 G M / c^{2}\right)$. Light speed rotating black holes can be called as "Special holes". Based on the classical force and power limits, the subject of current black hole physics can be simplified at fundamental level. Not only that the complicated mathematics of GTR can be refined.

\section{Acknowledgements}

Author is indebted to professor K. V. Krishna Murthy, Chairman, Institute of Scientific Research on Vedas (I-SERVE), Hyderabad, India and Shri K. V. R. S. Murthy, former scientist IICT (CSIR) Govt. of India, Director, Research and Development, I-SERVE, for their valuable guidance and great support in developing this subject.

\section{References}

[1] Ted Jacobson. Introductory lectures on black hole thermodynamics. 68 pages. www.physics.umd.edu/grt/taj/776b/lectures.pdf

[2] Hawking S.W. A brief history of time. Bantam Dell publishing group.1998.

[3] U.V.S. Seshavatharam, Physics of Rotating and Expanding Black Hole Universe, Progress in Physics, 2: 7-14, 2010.

[4] U.V.S. Seshavatharam. The Primordial Cosmic Black Hole and the Cosmic Axis of Evil. International Journal of Astronomy, 1(2): 20-37, 2012.

[5] Kirpal Nandra et al. The Growth of Super massive Black Holes Across Cosmic Time. http://constellation.gsfc.nasa.gov/documents/resources/presentations/Decadal/Nandra_AGN_GCT.pdf

[6] Christopher S. Reynolds. Astrophysics: Black holes in a spin. Nature. 494, 432-433 (28 February 2013) doi:10.1038/494432a

[7] Hawking, S. W. Black hole explosions?. Nature 248 (5443): 30. 1974.

[8] P.M. Alsing, P.W. Milonni. Simplified derivation of the Hawking-Unruh temperature for an accelerated observer in vacuum. American Journal of Physics 72 (12): 1524. 2004

[9] Hubble, E.P, The 200-inch telescope and some problems it may solve. PASP, 59: 153-167, 1947.

[10] U. V. S. Seshavatharam, S. Lakshminarayana and B. V. S. T. Sai. Is Red Shift-An Index of Galactic 'Atomic Light Emission' Mechanism?. International Journal of Physics, Vol. 1, No. 3, 49-64. 2013.

[11] Pathria, R. K. The Universe as a Black Hole. Nature 240 (5379): 298-299. 1972 doi:10.1038/240298a0

[12] Good, I. J. Chinese universes. Physics Today 25 (7): 15. July 1972. doi:10.1063/1.3070923.

[13] Poplawski, N. J. Radial motion into an Einstein-Rosen bridge. Physics Letters B 687 (2-3): 110-113. 2010.

[14] Zhang, Tianxi. Cosmic microwave background radiation of black hole universe. Astrophysics and Space Science, Volume 330, Issue 1, pp 157-165. 2010.

[15] Joel Smoller and Blake Temple. Shock-wave cosmology inside a black hole. Proc Natl Acad Sci U S A. September 30; 100(20): 1121611218. 2003.

[16] Chul-Moon Yoo et al. Black Hole Universe. Time evolution. arXiv:1306.1389v1 [gr-qc] 6 Jun 2013

[17] Victor Debattista et al. Disk Assembly and the M BH- $\sigma$ e Relation of Super massive Black Holes The Astrophysical Journal, Volume 765, Issue 1, article id. 23, 15 pp. 2013. http://www.uclan.ac.uk/news/black_holes_growing_faster_than_expected_new_study_shows.php

[18] Bennett, C.L.; Larson, L.; Weiland, J.L.; Jarosk, N.; Hinshaw, N.; Odegard, N.; Smith, K.M.; Hill, R.S. et al. Nine-Year Wilkinson Microwave Anisotropy Probe (WMAP) Observations: Final Maps and Results. arXiv:1212.5225. Retrieved December $22,2012$.

[19] Planck collaboration. Planck 2013 results. I. Overview of products and scientific results. Submitted to Astronomy \& Astrophysics. 2013. 
[20] Jian-Min Wang, Pu Du, David Valls-Gabaud, Chen Hu, and Hagai Netzer. Super-Eddington Accreting Massive Black Holes as Long-Lived Cosmological Standards, Physical Review Letters, Vol 110 Issue 8, 2013. DOI:10.1103/PhysRevLett.110.081301 